\title{
Fluorene Bilayer for Polymer Organic Light-Emitting Diode Using Efficient Ionization Method for Atomized Droplet
}

\author{
Takeshi Fukuda ${ }^{\mathrm{a}, *}$, Arata Sato ${ }^{\mathrm{a}, *}$ \\ ${ }^{a}$ Department of Functional Materials Science, Saitama University, 255 Shimo-Okubo, \\ Sakura-ku, Saitama 338-8570, Japan
}

\begin{abstract}
We present a solution-processed planar fluorene bilayer by an ultrasonic atomized deposition method in combination with a needle electrode as an ionization part for an atomized droplet. An important advantage of our method is that the atomized droplet is efficiently charged using a needle electrode, which speeds up the deposition rate of the polymer thin film. The deposition rate increases 2 to 3 times compared to a that obtained with a conventional technique without using the ionization method, and real-time monitoring of landed droplets indicates that the number of droplets increased as the voltage applied to the needle electrode was increased, owing to the highly charged atomized droplets. Furthermore, the TFB/F8BT bilayer was achieved by optimizing the substrate temperature, and the polymer organic light-emitting diode exhibits a luminance value exceeding $12,000 \mathrm{~cd} / \mathrm{m}^{2}$ by insertion of the TFB as an electron blocking layer. The maximum current efficiency of the fluorene bilayer device was $6.64 \mathrm{~cd} / \mathrm{A}$, which was a 3.2-fold increase compared to that obtained with the reference device without the TFB electron blocking layer.
\end{abstract}

Key words: Polymer organic light-emitting diode, Ultrasonic atomized deposition method, Needle electrode, Corona discharge, Ionization

${ }^{*}$ Corresponding author. Tel.: +81-48-858-3526; fax: +81-48-858-3526.
Email address: fukuda@fms.saitama-u.ac.jp (Takeshi Fukuda) 


\section{Introduction}

Polymer organic light-emitting diodes (P-OLEDs) have attracted interest for application in flat panel displays and general lighting owing to their high brightness, usage of large/flexible plastic substrate, very low fabrication cost because a roll-to-toll printing process is used, and tunable emission wavelength. The molecular structure and polymerization degree of polymer materials are important for improving the luminescence characteristics and long-term stability of P-OLEDs; therefore, novel polymers have been produced in previous studies [1-3]. In addition, a device structure is also investigated for achieving high device performance. For example, previous studies demonstrated superior high device performance using multilayer structures, inverted structures [4], and inserted electron/hole blocking layers to restrict the carrier recombination zone to the thin emissive layer [5]. However, the most serious problem of P-OLEDs is that it is difficult to fabricate the multilayer structure with a conventional solution process such as spray coating. This is because the underlying polymer layer is easily dissolved by the solvent during the coating of the upper polymer layer. Therefore, several chemical modification methods for the underlying layer and novel solution processes have been investigated for fabrication of the multilayer structure [6-9].

Currently, a poly (9,9-dioctylfluorene-alt- $N$-(4-butylphenyl)-diphenylamine)/poly(9,9-dioctylfluorene-alt-benzothiadiazole) (TFB/F8BT) bilayer is being investigated for improvement of the optical characteristics of P-OLED [10-13]. This is because the optimized fluorene polymer shows a high photoluminescence quantum yield of $>80 \%$ and high hole mobility $[14,15]$. These papers describe the reason for the improved performance of the bilayer device, namely: (i) reduced exciton quenching by shifting the carrier recombination zone away from the interface; (ii) reduced leakage of electrons from the F8BT emissive layer to the hole transport layer, resulting in efficient carrier confinement in the F8BT emissive layer. The energetic offset of the lowest occupied molecular orbital (LUMO) levels at the TFB/F8BT interface is $1.1 \mathrm{eV}$ [16], and this prevents electron leakage from the F8BT emissive layer to the hole transport layer [17]. As a result, the carrier recombination probability in the emissive layer increases, and this phenomenon is suitable for both high luminance efficiency and superior long-term stability even for P-OLEDs. In general, the TFB and F8BT bilayer are sometimes mixed at a heterojunction, and a mixed layer is formed. In the TFB:F8BT mixed layer, the primary exciton contribution is found to be $<2 \%$, which implies that $>98 \%$ of the 
excitons undergo charge transfer at the TFB/F8BT interface. Therefore, the relative PL quenching with respect to pure F8BT is only $<57 \%$ (PL yield of F8BT 80\%, of TFB:F8BT 35\%) [18]. For these reasons, the precious coating process for the TFB:F8BT bilayer has been attracting interest for P-OLED applications.

One approach is a transfer technique for fabricating the fluorene bilayer structure [19]. In this technique, the TFB layer is first prepared on the $\mathrm{O}_{2}$ plasma-treated glass substrate with a poly(styrenesulfonate) (PSS) layer to aid float-off in water. The F8BT-coated substrate is then laminated on the dry side of the aforementioned TFB-coated glass substrate. As a result, the TFB/F8BT bilayer can be formed without dissolving the underlying layer. However, the surface of the F8BT layer is relatively rough after removal of the PSS layer compared to those fabricated by other solution processes, and this will reduce the device performance. In another important technique, the non-orthogonal solvent used against the underlying layer (i.e., TFB) is used as the solvent for the upper layer (i.e., F8BT). In this case, the choices of polymer and solvent are limited, and the TFB/F8BT bilayer cannot be formed by solvent selection alone without modification of the chemical structure of F8BT. The solvent processing route makes scale-up feasible; however, concerns remain over the reproducibility of the entire process used in this method. Currently, a solution process called the electrospray deposition method has been investigated for fabricating the polymer multilayer structure. In this method, the highly charged droplets are continuously divided by Coulomb repulsion, and the small droplet size of $<1 \mu \mathrm{m}$ speeds up solvent evaporation [20]. Therefore, the multilayer structure can be realized [7-9]; however, the deposition rate is low approximately several $\mathrm{nm} / \mathrm{min}$, which results in low throughput of the mass production system. These disadvantages of previous studies prevented the practical use of solution-processed polymer-based electronic devices. Therefore, a simple fabrication process for the polymer multilayer has been an attractive research field.

Currently, we are investigating an ultrasonic atomized deposition method for fabricating a planar TFB/F8BT bilayer. Ultrasonic atomization methods have been investigated as a deposition method [21, 22]; however, the deposition efficiency is low, resulting in a low deposition rate of several $\mathrm{nm} / \mathrm{min}$ and low material utilization. Our methodology has advantages for several reasons such as the usage of an arbitrary solvent for the polymer, a simple experimental setup without the necessity of a vacuum chamber, and especially efficient ionization of droplets, which results in an improved deposition 
rate and lower surface roughness by solvent evaporation optimization [23].

In this study, we investigated an efficient ionization method for flying droplets in the ultrasonic atomized deposition method using the needle electrode. In this method, the corona discharge causes ionization of flying droplets, resulting in a high deposition efficiency corresponding to the high deposition rate. In addition, we demonstrate the TFB/F8BT bilayer structure and improved current efficiency of the multilayer P-OLED with TFB/F8BT.

\section{Experimental}

Figure 1 shows a schematic illustration of the ultrasonic atomized deposition method, which employs a needle electrode for ionization of atomized droplets. First, a polymer solution is continuously atomized by applying an ultrasonic wave to a polymer solution. The frequency of the ultrasonic wave was $3 \mathrm{MHz}$, and nitrogen gas $(0.5 \mathrm{~L} / \mathrm{min})$ was used as the carrier, which assists atomized droplet flow from the ultrasonic generator to the substrate. The most important part of this deposition method is the needle electrode, which was placed above the substrate. The diameter of the needle electrode was $20 \mathrm{~mm}$, which was larger than that of the glass tube. Therefore, the atomized droplets were not attached to the needle electrode. A high negative voltage of several kilovolts was applied to the needle electrode to ionize the flying droplets from the ultrasonic generator. As a result, the ionized droplets reach the substrate, resulting in the formation of a polymer thin film. In addition, the substrate temperature was controlled at $50{ }^{\circ} \mathrm{C}$ using a hot plate to optimize the speed of evaporation of the solvent [23]. The applied voltage was controlled from -1 to $-6 \mathrm{kV}$ at a fixed distance of $3.0 \mathrm{~cm}$ between the substrate and the needle electrode.

We investigated a fluorene bilayer, which contains TFB (ADS25913E, Mw $=10,000-200,000)$ and F8BT $(\mathrm{ADS} 133 \mathrm{YE}, \mathrm{Mw}=15,000-200,000)$ purchased from American Dye Source without further purification. Concentrations of TFB and F8BT were 1 and 0.1 wt $\%$ in $o$-dichlorobenzene (o-DCB), respectively. Because the TFB layer was formed by a spin-coating process, the concentration of the TFB solution is higher than that of the F8BT solution that was used in the ultrasonic atomized deposition method. First, the F8BT neat film was deposited on an indium tin oxide (ITO)-coated glass substrate $\left(R_{s}=15 \Omega / s q\right)$ for $10 \mathrm{~min}$. Then, the deposition rate and the surface roughness of the F8BT thin film were estimated by measuring the thickness and by using an atomic force microscope (AFM) image. In addition, a magnified 
image of a droplet that landed on a glass substrate was observed using an objective lens and a charge-coupled device camera (AUSB-K, Arms System). Next, the relative deposited area as a function of time was estimated using an image processing software (ImageJ), and the relative deposition area was calculated as the deposited area of the total droplet area divided by the observation area of the microscope image.

The device structure was ITO/PEDOT:PSS (40 nm)/TFB (25 nm)/F8BT $(70 \mathrm{~nm}) / \mathrm{LiF}(0.5 \mathrm{~nm}) / \mathrm{Al}(100 \mathrm{~nm})$ for the multilayer structure (device A), and the ITO/PEDOT:PSS (40 nm)/F8BT $(70 \mathrm{~nm}) / \mathrm{LiF}(0.5 \mathrm{~nm}) / \mathrm{Al}(100$ $\mathrm{nm}$ ) was used as a reference device (device B) without the electron blocking layer (EBL). The fabrication process for the multilayer OLED was as follows. First, an ITO-patterned glass substrate was cleaned for 20 min with ultrasonic cleaning using acetone, deionized water, and isopropyl alcohol. Next, the substrate was treated in the ultraviolet-ozone for 20 min. Poly(3,4ethylenedioxythiophene):polystyrene sulfonate (PEDOT:PSS; AI-4083, Clevious) was then spin-coated at a rotation speed of $2000 \mathrm{rpm}$ for $1 \mathrm{~min}$ on the ITO-patterned glass substrate as a hole transport layer. After annealing at $140{ }^{\circ} \mathrm{C}$ for $10 \mathrm{~min}$ in the atmosphere, TFB was also spin-coated in a nitrogen-purged grove box as an EBL. The rotation speed of the spin-coating process was $3000 \mathrm{rpm}$ for $1 \mathrm{~min}$. The thicknesses of the PEDOT:PSS and TFB layers were 40 and $25 \mathrm{~nm}$, respectively. The sample was then annealed at $130{ }^{\circ} \mathrm{C}$ for $20 \mathrm{~min}$, and the $\mathrm{F} 8 \mathrm{BT}$ thin film was deposited as an emissive layer with the ultrasonic atomized deposition method. A high voltage of -6 $\mathrm{kV}$ was applied to the needle electrode for depositing the F8BT layer, and this deposition condition was found to be optimized for achieving a fast deposition rate. The deposition time was $6 \mathrm{~min}$, and the thickness of the F8BT layer was $70 \mathrm{~nm}$. Finally, the sample was annealed at $70^{\circ} \mathrm{C}$ for $20 \mathrm{~min}$, after which the $\mathrm{LiF}(0.5 \mathrm{~nm}) / \mathrm{Al}(100 \mathrm{~nm})$ electrode was thermally deposited with a conventional thermal evaporation system.

The thickness was measured using a surface profiler (Alpha-Step IQ, KLA-Tencor). The surface roughness of the fabricated F8BT thin film was estimated using an AFM (Seiko, SPA-300). The depth profile of the atomic concentration was estimated using x-ray photoelectron spectrometry (XPS; Nova, KRATOS). The current density-voltage, luminance-voltage, and current efficiency-current density characteristics were measured using a DC voltage current source/monitor (6241A, ADCMT) and a luminance meter (LS100, Konicaminolta). 


\section{Results and discussion}

Figure 2(a) shows the relationship between the deposition rate of the F8BT neat film and the voltage applied to the electrode during the deposition process. Here, the needle and conventional mesh electrodes were used to compare the two electrodes. In the case of the needle electrode, the deposition rate continuously increased with increasing applied voltage up to $-6 \mathrm{kV}$, and the highest deposition rate of $10.5 \mathrm{~nm} / \mathrm{min}$ was achieved. During this process, corona discharge occurred at the tip of the needle electrode [24, 25]; therefore, flying droplets below the needle electrode were negatively charged upon applying the high voltage, as shown in Fig. 2(b), after which the ionized droplets were efficiently pulled toward the substrate, resulting in a fast deposition rate. This indicates that the higher voltage caused efficient ionization of the flying droplets [26], and the deposition rate increased with increasing voltage as shown in Fig. 2(a). However, a uniform F8BT thin film was not obtained at $-7 \mathrm{kV}$ owing to the very high deposition speed. The high deposition rate lowers the evaporation speed of $o$-DCB; therefore, a uniform F8BT thin film could not be deposited at $-7 \mathrm{kV}$ owing to the coffee stain phenomenon [27, 28]. On the other hand, in the case of the conventional mesh electrode [23], the deposition rate did not increase even though a high voltage was applied. This can be explained by the schematic configuration of the ionization image as shown in Fig. 2(c). In this experiment, the mesh electrode was inserted between the glass tube and the substrate instead of the needle electrode.

In general, the atomized droplets are negatively charged by the friction in the ultrasonic generator [29]. In addition, the flying droplets are also negatively charged by the application of a high voltage to the mesh electrode as shown in Fig. 2(c). Therefore, the Coulomb repulsion force causes the flying droplets to bounce before they pass through the mesh electrode. As a result, most of the droplets cannot be deposited on the substrate placed below the mesh electrode, resulting in a low deposition rate under the high-voltage condition, as shown in Fig. 2(a). On the other hand, in the case of the needle electrode, the flying droplets easily pass through the needle electrode even though a high voltage is applied. This is because the diameter of the needle electrode is larger than that of the glass tube, which determines the width of the droplet flow. The discharge amount of charge rapidly decreased with increasing distance from the electrode to the measurement point, and the charge density became $<0.01 \mathrm{nC}$, which is less than the minimum measure- 
ment range of the Coulomb meter (NK-1001A, Kasga Denki) when the high voltage of $-6 \mathrm{kV}$ was applied to the electrode. In the case of the needle electrode, the diameter is $20 \mathrm{~mm}$, therefore, the central part of atomized droplets are not ionized even though the high voltage was applied to the needle electrode. On the other hand, many metal wires were arranged in a reticular pattern in the case of the mesh electrode, the width of adjacent two metal wires were $2.5 \mathrm{~mm}$. This indicates that all of the atomized droplets were ionized by applying the high voltage to the mesh electrode before passing through the electrode. In addition, corona discharge efficiently occurred at the tip of the needle electrode [24, 25], and this phenomenon caused efficient ionization of the droplets after they passed through the mesh electrode. As a result, the well-charged droplets were efficiently deposited on the substrate, resulting in a high deposition rate by application of a high voltage in the case of the needle electrode.

To investigate the number of droplets deposited on the substrate, a microscope image of the landed droplets was observed during the deposition process. The needle electrode was used, and the applied voltage was changed to 0 and $-6 \mathrm{kV}$. In addition, the substrate was not treated with ultraviolet-ozone for clear observation of the droplet size. This is because the landed droplets rapidly spread on the ultraviolet-ozone-treated glass substrate owing to the small contact angle, and this hinders observation of the droplet shape. Figure 3(a) shows typical optical microscope images of the deposited droplets. These images clearly indicate that a large number of droplets were deposited when the voltage became high. In addition, relatively small droplets were deposited at $-6 \mathrm{kV}$. The most likely explanation is that the charged droplet is divided by Coulomb fission, as in electrospray deposition [30]. Next, the relative deposition area is calculated as the deposited area of the total droplet area divided by the observation area of the microscope image, using image processing software. Figure 3(b) shows the calculated relative deposited area as a function of time. This result also indicates that the relative deposited area increased with increasing voltage, and this result is in good agreement with the deposition rate, as shown in Fig. 2(a).

Figure 4 shows AFM images of F8BT neat films deposited with the ultrasonic atomized deposition method, and the applied voltage was changed from 0 to $-6 \mathrm{kV}$. In general, a balance between solvent evaporation and the deposition rate is an important factor for the formation of a uniform and smooth polymer thin film with the solution process [31]. Therefore, the evaporation speed of $o$-DCB should be controlled to realize a smooth thin film, and the 
substrate temperature was optimized as $50{ }^{\circ} \mathrm{C}$ against the vapor pressure of the solvent, which was $o$-DCB in this study [23]. Aggregated structures were observed when the voltage was low, that is, less than $-5 \mathrm{kV}$.

Figure 5(a) shows the relationship between the root-mean-square (RMS) roughness of the F8BT thin film estimated from the AFM image and the voltage applied to the needle electrode. Because the substrate temperature was optimized for the high deposition rate corresponding to the high voltage at $-6 \mathrm{kV}$, the RMS roughness decreased with increasing voltage, and the minimum RMS roughness of $3.7 \mathrm{~nm}$ was achieved at $-6 \mathrm{kV}$. This value is low enough to realize the multilayer OLED structure. To estimate this result, the evaporation time $\left(t_{e}\right)$ was calculated using the following equation [31]:

$$
t_{e}=\frac{d_{0}^{2}}{K_{e}(T)},
$$

where $d_{0}$ is the droplet size, which was estimated from the optical microscope image as shown in Fig. 3(a). In addition, $K_{e}(T)$ is the evaporation rate, which can be calculated from mass transfer models for an isolated spherical droplet. In this equation, the evaporation time of $o$-DCB was calculated for a spherical shape. However, the relative evaporation speed of $o$-DCB can be compared for discussing the relationship between the evaporation speed of $o$ DCB and the surface morphology of the F8BT thin film. $K_{e}(T)$ is calculated according to Eq. (2):

$$
K_{e}=8 D_{\text {diff }} \frac{\rho_{g}}{\rho} \operatorname{Ln}\left(\frac{1-Y_{\infty}}{1-Y_{s}}\right),
$$

where $D_{\text {diff }}$ is the mass diffusivity of the $o$-DCB molecule to the air, $\rho_{g}$ is the vapor density of $o$-DCB, $Y_{\infty}$ is the concentration of $o$-DCB far from the droplet, and $Y_{s}$ is the concentration of $o$-DCB at the droplet surface. $\rho_{g}, \rho$, and $P_{v} / P_{o}$ of $o-\mathrm{DCB}$ are $5.97 \mathrm{~kg} / \mathrm{m}^{3}, 1300 \mathrm{~kg} / \mathrm{m}^{3}$, and 0.00185 , respectively. $D_{\text {diff }}$ was calculated from Eq. (3) [32]:

$$
D_{\text {diff }}=0.0013 T^{1.5169}
$$

where $\mathrm{T}$ is the temperature around the droplet. The F8BT thin film was deposited at room temperature, and $\mathrm{T}$ was set at $298.15 \mathrm{~K}$. In addition, $Y_{s}$ can be calculated as $P_{v} / P_{0}$ (the ratio of vapor pressure of $o$-DCB to air). In fact, $Y_{\infty}$ is zero, and $Y_{s}=P_{v} / P_{0} \ll 1$; therefore Eq. (2) becomes 


$$
K_{e}=8 D_{\text {diff }} \frac{\rho_{g}}{\rho}\left(\frac{P_{v}}{P_{0}}\right)
$$

As a result, $t_{e}$ was calculated from equations (1)-(4).

Figure 5(b) shows the influence of the applied voltage on the droplet diameter of landed droplet on the substrate and the evaporation time of $o$-DCB. As mentioned earlier, the droplet size became small under the highvoltage condition. In general, the slow evaporation speed of the solvent causes the coffee stain phenomenon after droplet deposition, resulting in the formation of an aggregated structure and/or a nonuniform thin film [27, 28]. Therefore, a smooth surface was obtained with a shorter evaporation time, which is caused by the high voltage applied to the needle electrode.

An important advantage of this method is the small droplet size with a diameter of several $\mu \mathrm{m}$ as shown in Fig. 3(a), and this causes the high evaporation speed of the solvent. Therefore, the multilayer structure can be formed without dissolving the underlying organic layer. Both TFB (100 nm) and F8BT (100 nm) layers were continuously deposited on the substrate. After sample deposition, the depth profile of sulfur concentration was estimated from the XPS measurement result. Figure 6 shows the depth profile of sulfur concentration from the surface. This result clearly indicates that the concentration of sulfur was drastically reduced over $100 \mathrm{~nm}$. The molecular structures of TFB and F8BT are shown in the inset of Fig. 7. Only F8BT contains the sulfur molecule; therefore, this result indicates that the TFB/F8BT bilayer can be realized.

Figure 7 shows the cross-sectional view of the OLED with the TFB/F8BT bilayer. The structure of device A is ITO/PEDOT:PSS (40 nm)/TFB (25 $\mathrm{nm}) / \mathrm{F} 8 \mathrm{BT}(70 \mathrm{~nm}) / \mathrm{LiF}(0.5 \mathrm{~nm}) / \mathrm{Al}(100 \mathrm{~nm})$, and the TFB layer was removed as a reference device (device B). Because the TFB layer can be formed on the PEDOT:PSS layer by using a conventional spin-coating method, only the F8BT layer was deposited with the ultrasonic atomized deposition method. Figures $8(\mathrm{a})$ and $8(\mathrm{~b})$ show the current density-voltage and luminance-voltage characteristics of devices A and B, respectively. The turnon voltage was shifted toward the high voltage by inserting the TFB layer, which causes an energy barrier at the TFB/F8BT interface even though the hole mobility of TFB is greater than $10^{-3} \mathrm{~cm}^{2} / \mathrm{Vs}[33]$. The maximum luminance of device A, $12,200 \mathrm{~cd} / \mathrm{m}^{2}$, was higher than that of reference device $\mathrm{B}$ without the TFB layer $\left(4,450 \mathrm{~cd} / \mathrm{m}^{2}\right)$ owing to efficient carrier recombination in the F8BT layer. The higher current density of reference device B caused 
the breakdown of OLED at $9 \mathrm{~V}$. However, a voltage of $11.5 \mathrm{~V}$ could be applied to device A with the TFB/F8BT bilayer. Figure 9 shows the current efficiencies of devices $\mathrm{A}$ and $\mathrm{B}$; the current efficiency was greatly improved by inserting the TFB layer. The maximum current efficiencies of devices with and without the TFB layer were 6.8 and $2.0 \mathrm{~cd} / \mathrm{A}$, respectively. Therefore, the current efficiency was increased 3.4-fold by inserting the TFB layer as the EBL. In addition, the high current efficiency of device A was over 4.5 $\mathrm{cd} / \mathrm{A}$ at all the driving voltages. TFB influences the balance change in the bilayer device.

Figure 10 shows the energy diagram of device A. The LUMO level of TFB was $2.5 \mathrm{eV}$, which was lower than that of F8BT. Therefore, the injected electrons from the $\mathrm{Al}$ electrode are efficiently recombined in the F8BT emissive layer, which increases the current efficiency of the multilayer device as shown in Fig. 9.

\section{Conclusion}

In conclusion, we demonstrated a novel ionization method for the ultrasonic atomized deposition method, which is used to prepare a TFB/F8BT bilayer. By optimizing the applied voltage for the ionization process and the substrate temperature, the smooth surface of the F8BT layer is successfully deposited on the TFB thin film, and the evaporation speed of the solvent is found to affect the surface roughness. In addition, the TFB/F8BT bilayer was investigated with XPS measurement, and we demonstrated a 3.4-fold increase in the current efficiency of the multilayer OLED compared with the F8BT-only device.

\section{Acknowledgment}

This work was financially supported by JST A-STEP (AS2321387D). The authors thank Mr. Yoshitomi, Prof. Honda, and Prof. Kamata for their great help.

\section{References}

[1] J.H. Burroughes, D.D.C. Bradley, A.R. Brown, N. Marks, K. Mackay, R.H. Fried, P.L. Burns, A.B. Holmes, Light-emitting-diodes based on conjugated polymers, Nature, 347 (1990) 539-541. 
[2] X.Gong, J.C. Ostrowski, G.C. Bazan, D. Moses, A.J. Heeger, M.S. Liu, A.K.Y. Jen, Electrophosphorescence from a conjugated copolymer doped with an iridium complex: High brightness and improved operational stability, Adv. Mater., 15 (2003) 45-49.

[3] C. Sekine, Y. Tsubata, T. Yamada, M. Kitano, S. Doi, Recent progress of high performance polymer OLED and OPV materials for organic printed electronics, Sci. Technol. Adv. Mater., 15 (2014) 034203.

[4] M. Sessolo, H.J. Bolink, Hybrid Organic-Inorganic Light-Emitting Diodes, Adv. Mater., 23 (2011) 1829-1845.

[5] S. Reineke, M. Thomschke, B. Lussem, K. Leo, White organic lightemitting diodes: Status and perspective, Rev. Mod. Phys., 85 (2013) 1245 .

[6] N. Aizawa, Y.-J. Pu, M. Watanabe, T. Chiba, K. Ideta, N. Toyota, M. Igarashi, Y. Suzuri, H. Sasabe, J. Kido, Solution-processed multilayer small-molecule light-emitting devices with high-efficiency whitelight emission, Nature Commun., 5 (2014) 5756.

[7] Y. Koishikawa, H. Miyazaki, M. Yahiro, C. Adachi, Multi-layered organic light-emitting diode fabrication using low molecular weight materials by electrospray method, Thin Solid Films, 31 (2013) 527-532.

[8] M. Ali, M. Abbas, S. K. Shah, R. Tuerhong, A. Generosi, B. Paci, L. Hirsch, and R. Gunnella, Realization of solution processed multi-layer bulk heterojunction organic solar cells by electro-spray deposition, Org. Electron., 13 (2012) 2130-2137.

[9] T. Fukuda, K. Takagi, Y. Liao, Insertion of fullerene layer for bulk heterojunction organic photovoltaic cell fabricated by electrospray deposition method, Phys. Status Sol.: Rapid Research Lett., 7 (2013) 1055-1058.

[10] J.-S. Kim, R.H. Friend, I. Grizzi, J.H. Burroughes, Spin-cast thin semiconducting polymer interlayer for improving device efficiency of polymer light-emitting diodes, Appl. Phys. Lett., 87 (2005) 023506.

[11] T. Chiba, Y.-J. Pu. M. Hirasawa, A. Masuhara, H. Sasabe, J. Kido, Solution-Processed Inorganic-Organic Hybrid Electron Injection Layer 
for Polymer Light-Emitting Devices, ACS Appl. Mater. Interfaces, 4 (2012) 6104-6108.

[12] J.C.D. Faria, A.J. Campbell, M.A. McLachlan, Fluorene copolymer bilayers for emission colour tuning in inverted hybrid light emitting diodes, J. Mater. Chem. C, 3 (2015) 4945-4953.

[13] K.-H. Yim, Z. Zheng, Z. Liang, R.H. Friend, W.T.S. Huck, J.-S. Kim, Efficient Conjugated-Polymer Optoelectronic Devices Fabricated by ThinFilm Transfer-Printing Technique, Adv. Funct. Mater., 18 (2008)10121019 .

[14] A.J. Cadby, R. Dean, C. Elliott, R.A.L.Jones, A. Mark Fox, D.G. Lidzey, Imaging the Fluorescence Decay Lifetime of a Conjugated-Polymer Blend By Using a Scanning Near-Field OpticalMicroscope, Adv. Mater., 19 (2007) 107-111.

[15] L.-L. Chua, J. Zaumseil, J.-F. Chang, E. C.-W. Ou, P. K.-H. Ho, H. Sirringhaus, and R.H. Friend, General observation of n-type field-effect behaviour in organic semiconductors, Nature, 434 (2005) 194-199.

[16] T.A. Skotheim. Handbook of Conducting Polymers, Third Edition. CRC Press, 2006.

[17] J. Bailey, E.N. Wright, X. Wang, A.B. Walker, D.D.C. Brandley, J.S. Kim, Understanding the role of ultra-thin polymeric interlayers in improving efficiency of polymer light emitting diodes, J. Appl. Phys., 115 (2014) 204508.

[18] A.C. Morteani, P. Sreearunothai, L.M. Herz, R.H. Friend, C. Silva, Exciton Regeneration at Polymeric Semiconductor Heterojunctions, Phys. Rev. Lett., 92 (2004) 247402.

[19] D. Kabra, M.H. Song, B. Wenger, R.H. Friend, H.J. Snaith, High Efficiency Composite Metal Oxide-Polymer Electroluminescent Devices: A Morphological and Material Based Investigation, Adv. Mater., 20 (2008) 2447-2452.

[20] A.M. Gañán-Calvo, J. Dávila, A. Barrero, Current and droplet size in the electrospraying of liquids. Scaling laws, J. Aerosol Sci., 28 (1997) 249-275. 
[21] X.X. Steirer, M.O. Reese, B.L. Rupert, N. Kopodakis, D.C. Olson, R.T. Collins, D.S. Ginley, Ultrasonic spray deposition for production of organic solar cells, Solar Energy Mater. Solar Cells, 93 (2009) 447-453.

[22] K. Gilissen, J. Stryckers, P. Verstappen, J. Drikoningen, G.H.L. Heintges, L. Lutsen, J. Manca, W. Maes, W. Deferme, Ultrasonic spray coating as deposition technique for the light-emitting layer in polymer LEDs, Org. Electron., 20 (2015) 31-35.

[23] A. Sato, T. Fukuda, N. Kamata, Z. Honda, Efficient ionization method of atomized droplet for fabricating multilayer organic light-emitting diode, Proceeding of the 21st International Display Workshops (2014) 763-766.

[24] J.-C. Wang, D. Zhang, N. Keoni, H. Birecki, O. Gila, M.J. Kushner, Charging of moving surfaces by corona discharges sustained in air, J. Appl. Phys., 116 (2014) 043301.

[25] A Mraihi, N Merbahi, M Yousfi, A Abahazem and O Eichwald, Electrical and spectroscopic analysis of mono- and multi-tip pulsed corona discharges in air at atmospheric pressure, Plasma Sources Sci. Technol., 20 (2011) 065002.

[26] M. Lackowski, A. Krupa, A. Jaworek, Corona discharge ion sources for fine particle charging, Eur. Phys. J. D, 56 (2010) 377-382.

[27] G.-H. Lim, J.-M. Zhuo, L.-Y. Wong, S.-J. Chua, L.-L. Chua, P.K.H. Ho, A transition solvent strategy to print polymer:fullerene films using halogen-free solvents for solar cell applications, Org. Electron., 15 (2014) 449-460.

[28] T. Kajiya, D. Kaneko, M. Doi, Dynamical Visualization of "Coffee Stain Phenomenon" in Droplets of Polymer Solution via Fluorescent Microscopy, Langmuir, 24 (2008) 12369-12374.

[29] E.E. Dodd, The statistics of liquid spray and dust electrification by the Hopper and Laby method, J. Appl. Phys., 24 (1953) 73-80.

[30] K.Y. Li, H.H. Tu, A. K. Ray, Charge Limits on Droplets during Evaporation, Langmuir, 21 (2005) 3786-3794. 
[31] X.-Y. Zhao, W. Yang, C. Li, X. Wang, S.L. Lim, D. Qi, R. Wang, Z.-Q. Gao, B.-X. Mi, Z.-K. Chen, W. Huang, W. Deng, Effects of Damkhöler number of evaporation on the morphology of active layer and the performance of organic heterojunction solar cells fabricated by electrospray method, Solar Mater. Solar Cells, 134 (2015) 140-147.

[32] B. Abramzon, W.A.Sirignano, Droplet vaporization model for spray combustion calculations, Int. J. Heat Mass Transfer, 32 (1989) 16051618.

[33] M. Redecker, D.D.C. Bradley, M. Inbasekaran, W.W. Wu, E.P. Woo, High Mobility Hole Transport Fluorene-Triarylamine Copolymers, Adv. Mater., 11 (1999) 241-246. 


\section{Figure captions}

Figure 1 Experimental setup of ultrasonic atomized deposition method in combination with needle electrode. Inset shows photograph of needle electrode having $20 \mathrm{~mm}$ diameter.

Figure 2 (a) Relationship between the applied voltage to the needle electrode and the deposition rate of F8BT thin film for both Relationship between voltage applied to needle electrode and deposition rate of F8BT thin film for both cases: needle electrode and mesh electrode. Schematic images of droplet flow for (b) needle electrode and (c) mesh electrode.

Figure 3 (a) Typical optical microscope images of droplets deposited on ITO-coated glass substrate without ultraviolet-ozone treatment at different voltages and deposition times. (b) Relative deposited area as function of deposition time. Voltage applied to needle electrode was changed from 0 to $-6 \mathrm{kV}$ to estimate effect of ionization phenomena on deposition rate.

Figure 4 AFM images of F8BT thin films deposited at different applied voltages. Applied voltages were (a) -1, (b) -2, (c) -3, (d) -4, (e) -5, and (f) $-6 \mathrm{kV}$.

Figure 5 (a) Relationship between the RMS roughness of F8BT thin film and the voltage applied to needle electrode. (b) Droplet diameter and evaporation time of $o-\mathrm{DCB}$ as a function of voltage applied to needle electrode.

Figure 6 Depth profile of sulfur concentration for multilayer structure with planar F8BT (100 nm)/TFB (100 nm) bilayer deposited by ultrasonic atomized deposition method.

Figure 7 Cross-sectional view of device A with TFB/F8BT bilayer and molecular structures of F8BT and TFB.

Figure 8 (a) Current density-voltage and (b) luminance-voltage characteristics of devices $\mathrm{A}$ and $\mathrm{B}$ with and without TFB layer used as EBL.

Figure 9 Current efficiency-current density characteristics of OLEDs with and without TFB EBL.

Figure 10 Energy diagram of OLED with TFB/F8BT bilayer (device A). 


\section{FIGURES}

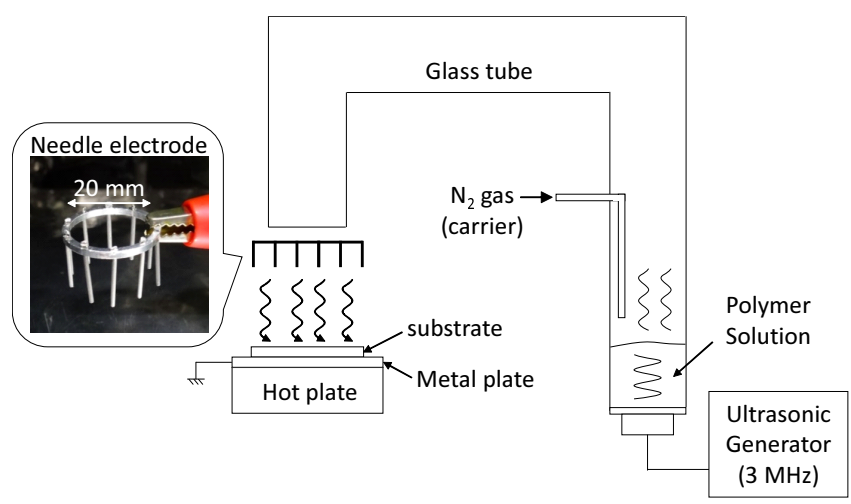

Figure 1: Experimental setup of ultrasonic atomized deposition method in combination with needle electrode. Inset shows photograph of needle electrode having $20 \mathrm{~mm}$ diameter. 


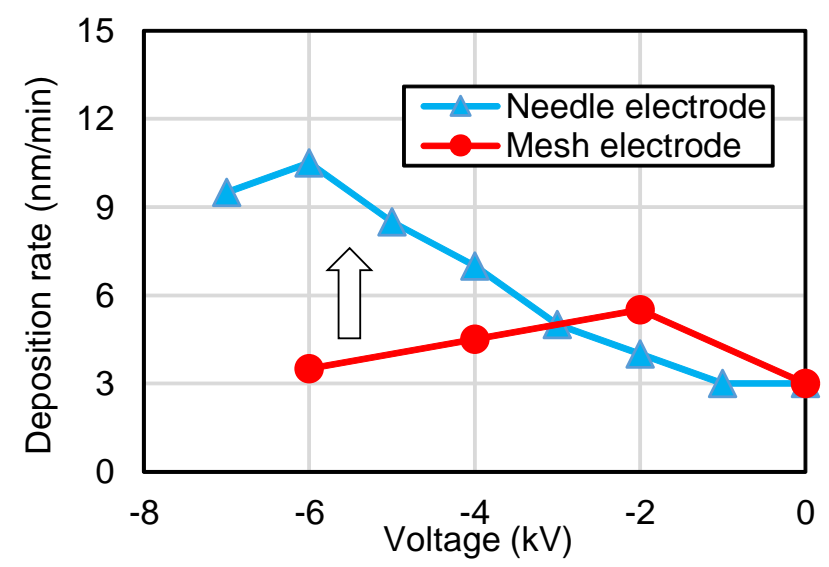

(a)

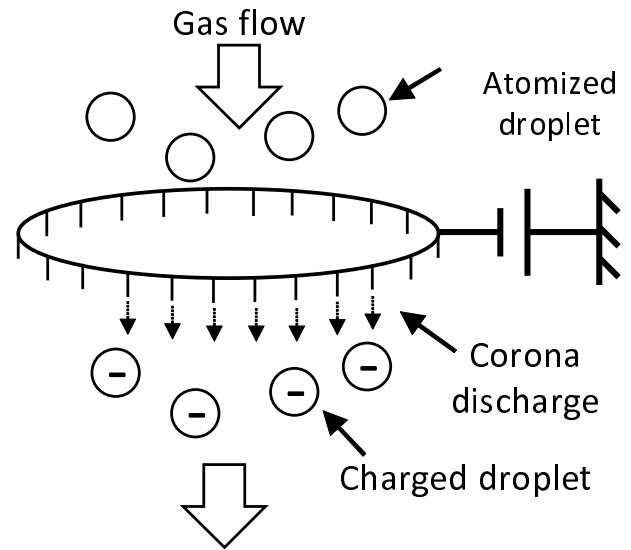

(b)

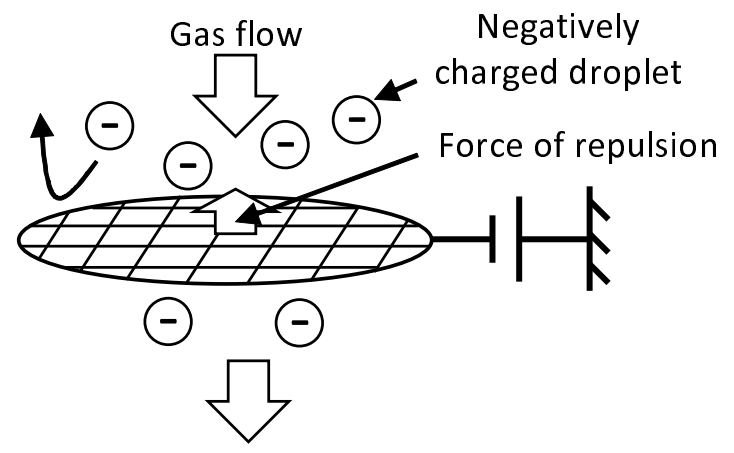

(c)

Figure 2: (a) Relationship between the applied voltage to the needle electrode and the deposition rate of F8BT thin film for both Relationship between voltage applied to needle electrode and deposition rate of F8BT thin film for both cases: needle electrode and mesh electrode. Schematic images of droplet flow for (b) needle electrode and (c) mesh electrode. 


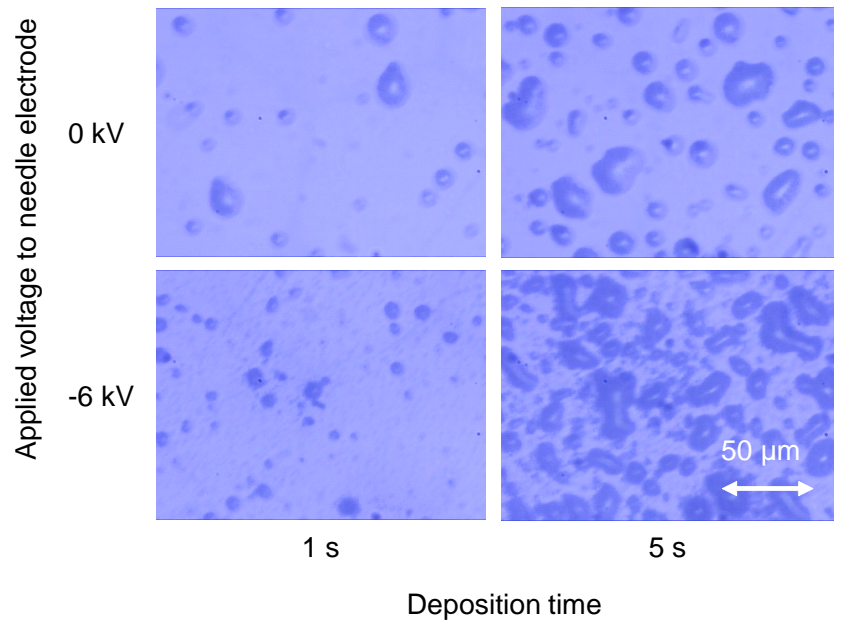

(a)

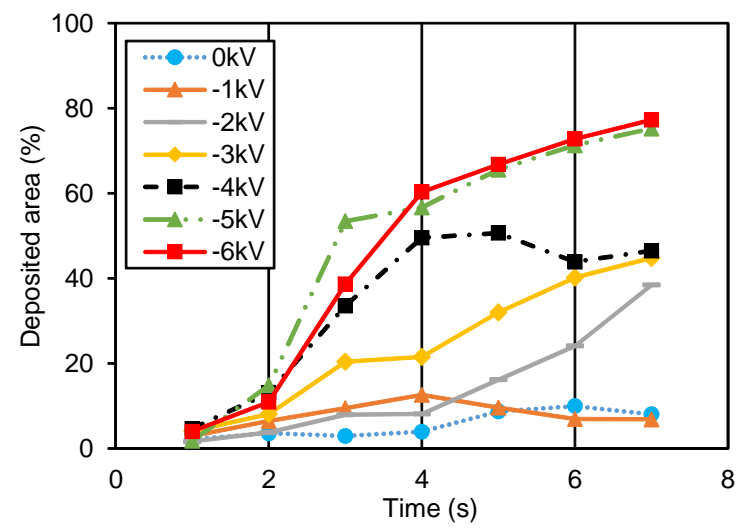

(b)

Figure 3: (a) Typical optical microscope images of droplets deposited on ITO-coated glass substrate without ultraviolet-ozone treatment at different voltages and deposition times. (b) Relative deposited area as function of deposition time. Voltage applied to needle electrode was changed from 0 to $-6 \mathrm{kV}$ to estimate effect of ionization phenomena on deposition rate. 


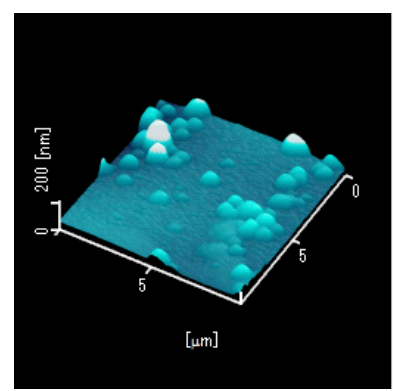

(a)

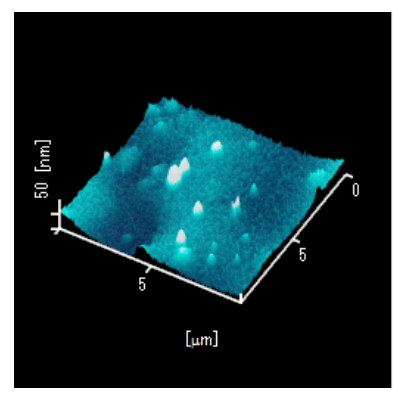

(d)

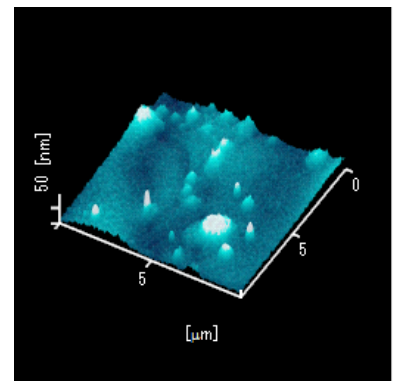

(e)

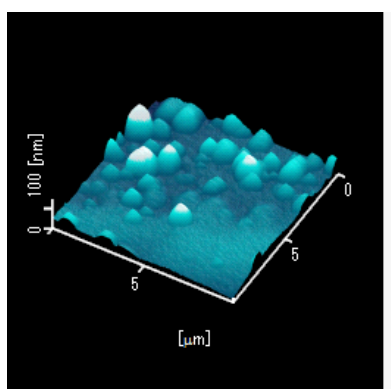

(b)

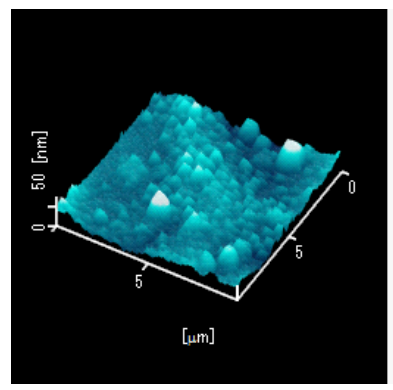

(c)

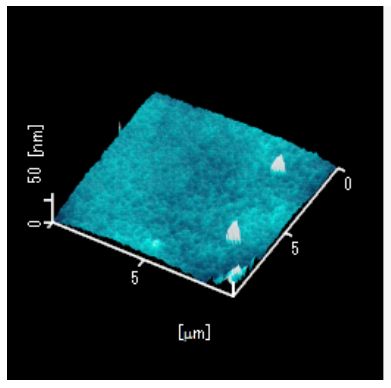

(f)

Figure 4: AFM images of F8BT thin films deposited at different applied voltages. Applied voltages were (a) -1 , (b) -2 , (c) -3 , (d) -4 , (e) -5 , and (f) $-6 \mathrm{kV}$. 


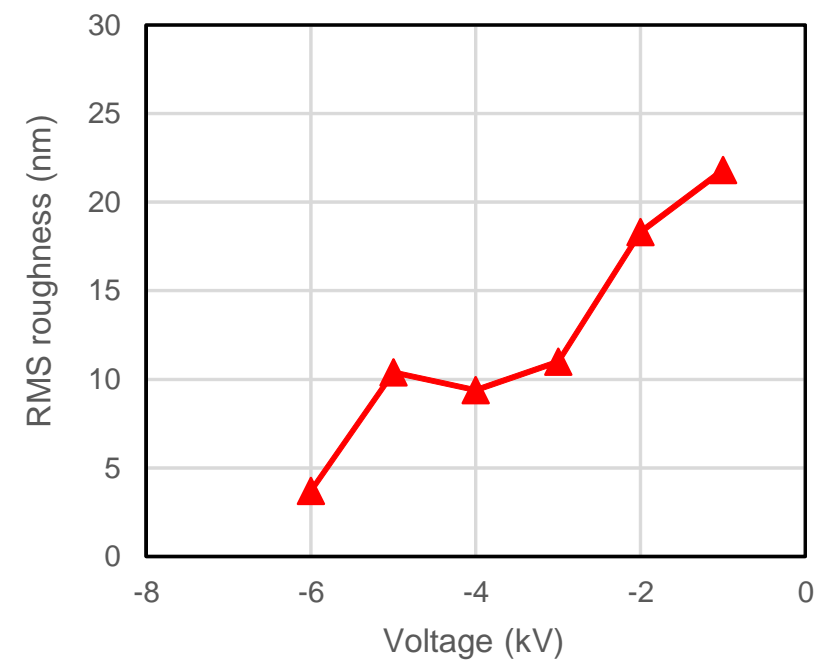

(a)

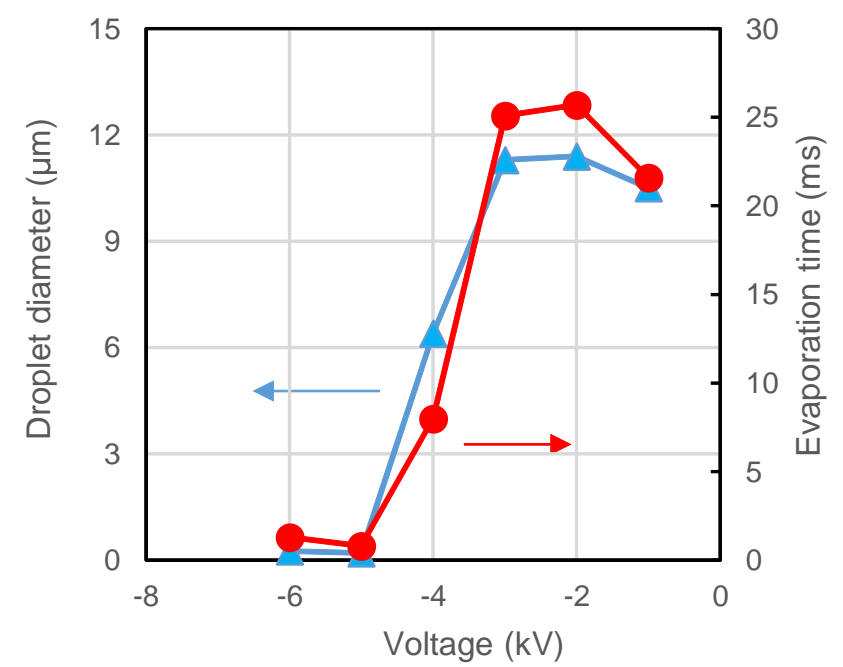

(b)

Figure 5: (a) Relationship between the RMS roughness of F8BT thin film and the voltage applied to needle electrode. (b) Droplet diameter and evaporation time of $o$-DCB as a function of voltage applied to needle electrode. 


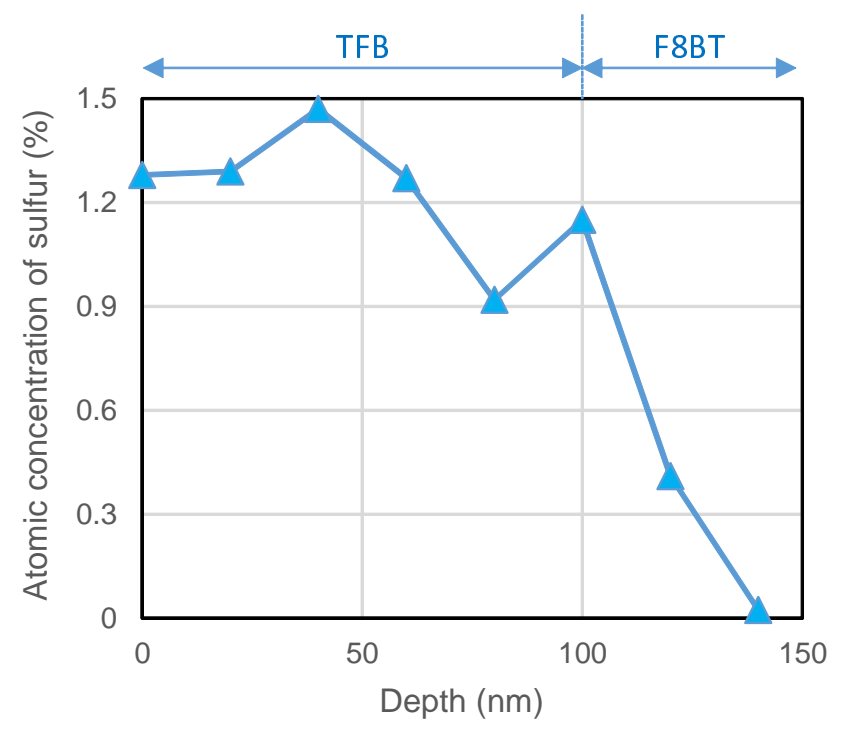

Figure 6: Depth profile of sulfur concentration for multilayer structure with planar F8BT $(100 \mathrm{~nm}) /$ TFB $(100 \mathrm{~nm})$ bilayer deposited by ultrasonic atomized deposition method.

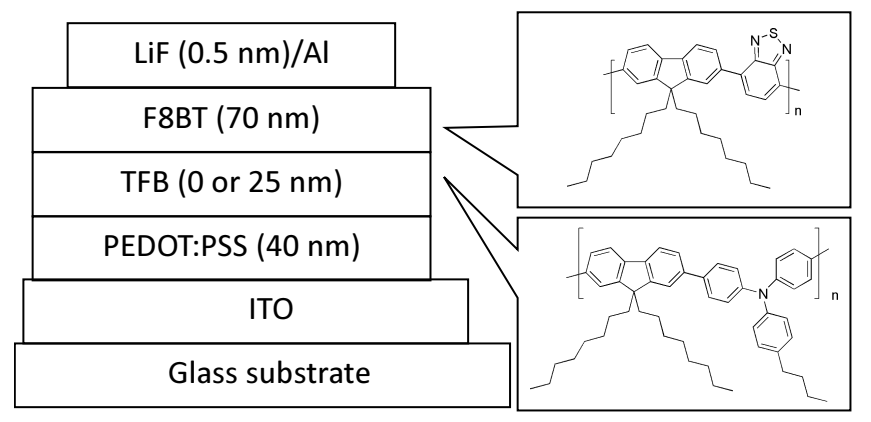

Figure 7: Cross-sectional view of device A with TFB/F8BT bilayer and molecular structures of F8BT and TFB. 


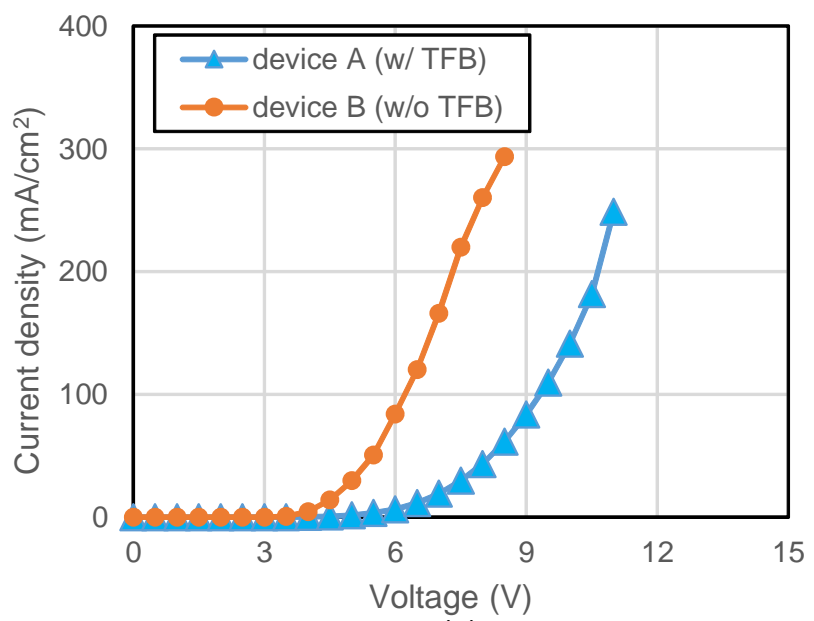

(a)

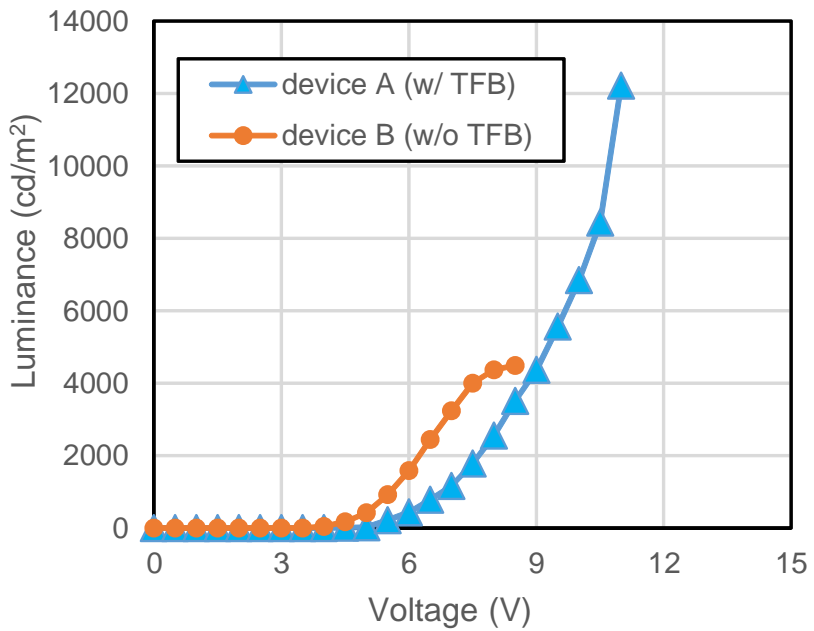

(b)

Figure 8: (a) Current density-voltage and (b) luminance-voltage characteristics of devices $\mathrm{A}$ and $\mathrm{B}$ with and without TFB layer used as EBL. 


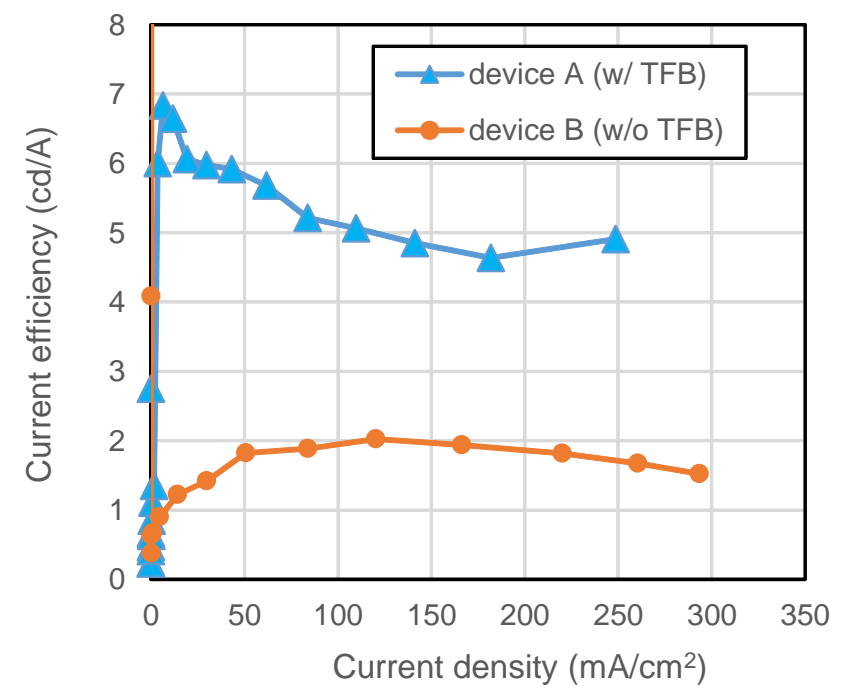

Figure 9: Current efficiency-current density characteristics of OLEDs with and without TFB EBL.

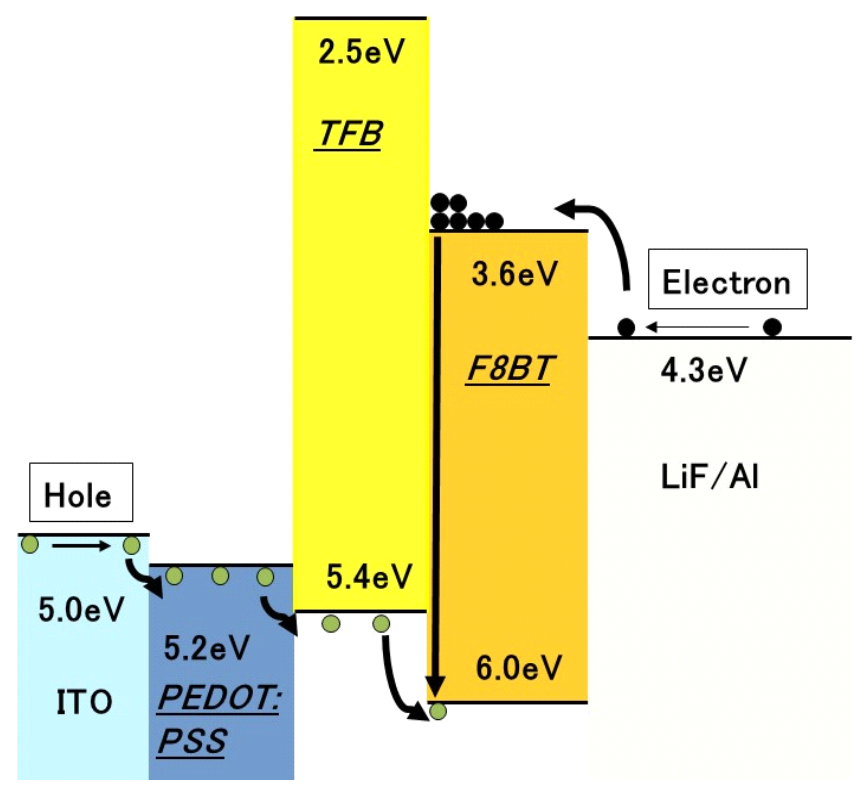

Figure 10: Energy diagram of OLED with TFB/F8BT bilayer (device A). 

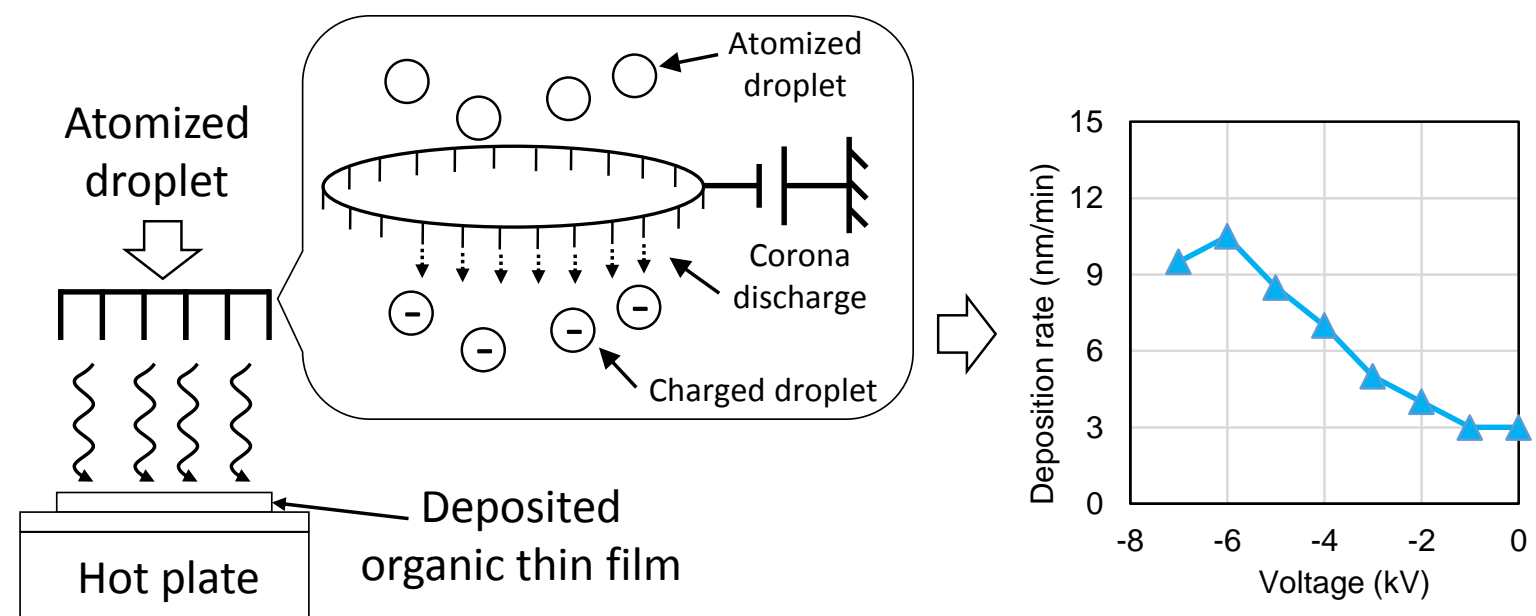